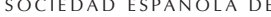 Cerámica y Vidrio $\begin{array}{lllllllllllllllll}\text { A } & \mathrm{R} & \mathrm{T} & \mathrm{I} & \mathrm{C} & \mathrm{U} & \mathrm{L} & \mathrm{O}\end{array}$
}

\section{Caracterización de ladrillería tradicional producida en la Vega del Guadalquivir, en zonas próximas a Sevilla.}

\author{
*V. FLORES ALÉS, ** A. GUIRAÚM PÉREZ, Y*** J. BARRIOS SEVILLA. \\ * DPTO. CONSTRUCCIONES ARQUITECTÓNICAS II. E.U.ARQUITECTURA TÉCNICA, SEVILLA. \\ ** DPTO. QUÍMICA ANALÍTICA. \\ *** DPTO. CONSTRUCCIONES ARQUITECTÓNICAS I. UNIVERSIDAD DE SEVILLA
}

\begin{abstract}
En el presente trabajo se han establecido las características físicas, químicas y mineralógicas de un grupo de piezas procedentes de las ladrilleras situadas en la vega del río Guadalquivir, en la zona situada entre Sevilla, Coria, San Juan de Aznalfarache, Tomares y Castilleja de la Cuesta, y que continúan funcionando con un sistema de producción latericia tradicional, similar a la que se ha venido empleando desde hace siglos. Las variaciones que presenta el proceso de fabricación con respecto a la técnica de elaboración seguida desde hace siglos se limitan casi exclusivamente a los combustibles utilizados en los hornos de cocción.

La caracterización de los materiales se ha realizado mediante el estudio de propiedades físicas mediante ensayos normalizados, análisis químico de componentes mayoritarios y estudio estadístico de resultados, análisis mineralógico por difracción de rayos X con valoración semicuantitativa de resultados y análisis térmico mediante ATD y termogravimetría.

Las piezas estudiadas se han separado inicialmente en dos grupos, según la coloración amarilla o rojiza de la pasta cerámica, presentando el primero una textura más fina producto de la materia prima; estas características diferenciadoras descritas a priori se han visto parcialmente confirmadas por los resultados obtenidos; lógicamente las composiciones químicas son diferentes, las piezas rojizas presentan una mayor resistencia a compresión, mientras que los ensayos de succión y absorción son similares. Independientemente de la influencia de las transformaciones que se producen durante la cocción en la coloración, en la composición mineralógica no se aprecian diferencias sustanciales entre los dos grupos (1).

Se ha comprobado la homogeneidad y la correlación existente en los distintos parámetros analizados en función del análisis estadistico factorial, así como entre ellos; y se han establecido las temperaturas alcanzadas durante la cocción en función de la presencia de minerales sintéticos en la composición mineralógica. Los resultados de los ensayos físicos y las diferencias existentes en el dimensionado de las piezas están acordes con el proceso manual seguido y las deficiencias del proceso de fabricación.
\end{abstract}

Palabras clave: Sevilla, margas, ladrillo, análisis factorial.

Characterization of traditional bricks from the Vega del Guadalquivir, in the zones near Seville.

In this work it has been established pyshical, chemical and mineralogical characteristics of a group of pieces from bricks manufactures situated in the Guadalquivir river banks, in the zone between the city of Seville and the towns of Coria, San Juan de Aznalfarache, Tomares and Castilleja de la Cuesta, which continue working with traditional production systems, as they have been made from centuries. The differences that the processes show respect old traditional techniques are limited to the combustibles utilized in the firing kilns.

Samples characterization were done by the study of physical properties according to quality standards tries, chemical analysis of main components and stadistic study of the results, mineralogical qualitative and semicuantitative analysis by $\mathrm{X}$ ray diffraction and thermal analysis by DTA and thermogravimetry.

The pieces of this study were initially classified in two groups according to the paste colour (red or yellow). The yellow one present a fine grain texture related with the raw materials; differential characteristics observed were partially confirmed by the results obtained. Chemical compositions are different, red pieces show a higher mechanical compression resistance, while suction and absortion values are similar. Mineralogycal composition does not show important differences between groups (1).

It have been confirmed the homogenity in the parameters analyzed and the relationships between them, from the statistical factorial analysis. Temperatures reached in the firing process have beeen established according to the syntetic minerals detected in the mineralogical analysis. Physical tries results and morfological differences are due to the manual moulding process and irregular manufacturing system.

Key words Seville, marls, brick, factor analysis.

\section{INTRODUCCIÓN}

El presente trabajo se enmarca dentro de un estudio de los ladrillos producidos en las diversas comarcas de la provincia de Sevilla por métodos tradicionales. En la actualidad aún siguen existiendo muchos tejares que fabrican sus productos con una tecnología muy rudimentaria, produciendo un material de características muy rústicas y aplicaciones muy concretas, fundamentalmente elementos decorativos y para pavimentos.

En este caso se han realizado estudios físicos, químicos, mineralógicos y térmicos sobre un grupo de piezas de dos tipos distintos, procedentes de las ladrilleras ubicadas junto al río Guadalquivir en la zona situada entre las localidades San Juan de Aznalfarache y Camas, en la zona conocida como La Pañoleta (Figura 1). En dichas ladrilleras se siguen elaborando las piezas a mano, con proceso tradicional de amasado y moldeado en gradilla de madera sobre el suelo, previamente espolvoreado con cal, y cocción en hornos discontinuos.

Toda esta zona de la provincia de Sevilla, situada en la comarca conocida como La Vega, esta compuesta por depósitos del Cuaternario que forman las terrazas del río; en la zona próxima a la capital estos depósitos están compuestos básicamente por cantos rodados, arenas y arcillas rojizas y pardas 
$(2,3)$. En general los alrededores de la ciudad de Sevilla son muy heterogéneos en su litología, como sedimentos propios de terrazas. Conforme se produce el alejamiento hacia el oeste, desde la margen derecha del cauce, se pueden encontrar los afloramientos de materiales del Terciario (4), margas marrones y arenas, siendo las margas azules más abundantes hacia el norte y los limos arenosos hacia el sur; ambas litologías tienen gran continuidad (5).

La vega del Guadalquivir a su paso por Sevilla ha sido, desde siempre, una zona en la que han proliferado tejares y alfares que han venido utilizando materiales arcillosos mezclados con margas azules que afloran en la margen derecha del río, como se indica anteriormente. La zona en la que están situadas las explotaciones de arcillas para cerámica es la terraza más reciente del río, rica en arcillas oscuras y gravas. Se distinguen dos tipos de arcillas, unas grasas, más compactas y que forman terrones, de color rojizo, y otras arenosas de color más claro, ambas contienen un elevado porcentaje de carbonato cálcico.

Los materiales que tradicionalmente se extraían en la zona concreta de la que proceden las muestras reciben el nombre de "barros azules de La Pañoleta". Estos barros están compuestos por margas azules del Plioceno (6), muy plásticas, limos y arcillas del Cuaternario de naturaleza illítica y montmorillonítica, presentando vetas de óxidos de hierro. Los procedentes de la zona próxima a Coria del Río están constituidos también por sedimentos aluviales de similares características y presencia sensible de carbonato de calcio. Estos materiales son objeto de expolotación para la fabricación de diferentes productos cerá$\operatorname{micos}(7,8)$

\section{MATERIALES Y MÉTODOS.}

El grupo de piezas estudiadas se ha dividido en dos subgrupos de quince muestras cada uno de ellos, atendiendo al color de la pasta base, distinguiéndose un grupo de piezas de color rojizo, y otro de color amarillo, de esta manera, las muestras se han signado como PR y PA respectivamente. Por lo general todas las piezas presentan mala apariencia y mal acabado, sobre todo en una de sus tablas. Estos pequeños tejares trabajan a partir de materias primas procedentes en su mayoría de las zonas que se extiendan hasta la localidad cercana de Coría del Río debido a la escasez de materiales de calidad en la zona provocada por la extracción masiva desde hace siglos.

Las distintas técnicas analíticas seguidas en función del tipo de análisis fueron las siguientes:

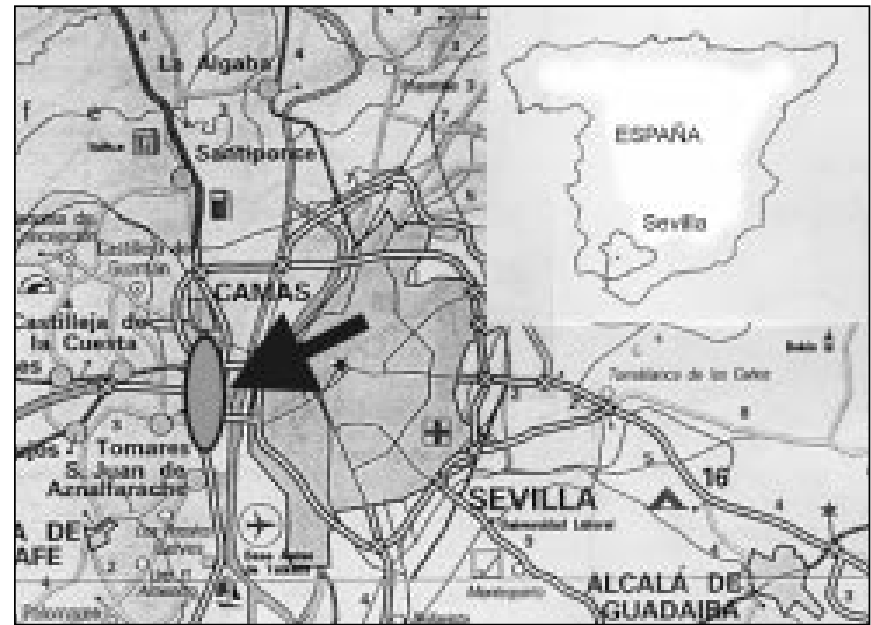

Fig. 1. Mapa de situación de la ciudad de Sevilla y zonas de extracción de materias primas y fabricación de materiales cerámicos.

En los ensayos físicos se llevaron a cabo tres tipos de estudios, absorción y succión de agua y resistencia a compresión, en los tres casos se siguieron las prescripciones indicadas por las normas UNE de control de calidad para ladrillos cerámicos actualmente vigentes (UNE 67-025, UNE 67-031, y UNE 67-026)

Los análisis químicos de componentes mayoritarios se realizaron en fase líquida atendiendo a la naturaleza del analito. Las técnicas utilizadas fueron: espectroscopía de plasma ICP, en equipo Fisons 3410-1461, para el análisis de $\mathrm{Al}, \mathrm{Fe}, \mathrm{Ca}, \mathrm{Mg}$, Ti, Na y K, turbidimetría por espectrofotometría UV/visible, en espectrofotómetro Phillips PU 8720, para la determinación del contenidos en sulfatos, determinándose la pérdida por calcinación (P.F.) a $1000^{\circ} \mathrm{C}$ y cuantificándose la sílice mediante la fusión alcalina de la muestra y posterior precipitación en medio ácido. Las muestras fueron diluidas mediante ataque ácido ( $\mathrm{HF}, \mathrm{HClO}_{4}$ y agua regia) en caliente (9).

Los resultados obtenidos han sido tratado estadísticamente mediante análisis factorial desarrollado en base a los parámetros más significativos, tratando de establecer diferencias significativas entre los diferentes grupos de muestras, este se ha llevado a cabo con el programa informático de tratamiento de resultados "statistica" (StatSoft, Inc. 1993). Este análisis ha permitido establecer tres factores que engloban cinco parámetros químicos con significación propia y que discriminan de manera clara los dos grupos.

El estudio de la composición mineralógica se realizó por

TABLA I

Resultados de ENSAYOS Físicos

\begin{tabular}{|l|l|l|l|l|l|}
\hline muestras & ensayos/valores & mínimo & máximo & media & desv.std. \\
\hline PR & Succión $\left(\mathrm{gr} / \mathrm{cm}^{2}\right)$ & 0.38 & 0.22 & 0.29 & 0.043 \\
& Absorción $(\%)$ & 31.1 & 22.9 & 27.6 & 2.8 \\
& Res.Comp. $\left(\mathrm{Kp} / \mathrm{cm}^{2}\right)$ & 282.8 & 222.9 & 237.3 & 18.0 \\
\hline PA & Succión $\left(\mathrm{gr} / \mathrm{cm}^{2}\right)$ & 0.39 & 0.25 & 0.31 & 0.036 \\
& Absorción $(\%)$ & 27.8 & 22.2 & 27.1 & 0.5 \\
& Res.Comp. $\left(\mathrm{Kp} / \mathrm{cm}^{2}\right)$ & 218.3 & 193.9 & 204.7 & 5.1 \\
\hline
\end{tabular}


difracción de rayos $\mathrm{X}$ mediante método de polvos, en difractómetro Phillips PW 1130/90 dotado de rendija semiautomática, empleando la radiación $\mathrm{K} \alpha$ del $\mathrm{Cu}$ con filtro de $\mathrm{Ni}$ en el intervalo de $3^{\circ}$ a $7 \Theta^{\circ}$, a una velocidad de $2^{\circ} 2 / \mathrm{min}$, con posterior evaluación semicuantitativa en función de los poderes de reflectantes y altura de los picos fundamentales.

Los análisis térmicos, diferencial y gravimétrico, se llevaron a cabo en equipo de alta temperatura, en atmósfera inerte de nitrógeno con termopar de $\mathrm{Pt} / \mathrm{Pt}$-Rh $(13 \%)$ a partir de una referencia de alúmina calcinada, estando las muestras preparadas con un tamaño de grano inferior a $50 \mu \mathrm{m}$ y llevando estas hasta $1000^{\circ} \mathrm{C}$ a una velocidad de calentamiento de $10^{\circ} \mathrm{C} / \mathrm{min}$ y posterior enfriamiento a $50^{\circ} \mathrm{C} / \mathrm{min}$.

\section{RESULTADOS Y DISCUSIÓN.}

\subsection{Ensayos físicos.}

Los dos grupos de ladrillos estudiados no presentan divergencias apreciables en los resultados de succión y absorción de agua, lo que manifiesta un proceso de amasado y moldeo similar. Todos los valores de succión, aún siendo elevados, están por debajo de los mínimos de calidad según norma UNE $\left(0.45 \mathrm{~g} / \mathrm{cm}^{2}\right)$. No ocurre lo mismo con la absorción, cuyos resultados sobrepasan con creces el 18\%. En general, los datos obtenidos exponen una desviación de resultados muy pequeña.

A diferencia de los resultados manifestados para los ensayos no destructivos, los valores de resistencia a compresión presentan diferencias entre los dos grupos, ya que las piezas rojas tienen un mejor comportamiento, todos los resultados se encuentran muy por encima de los $100 \mathrm{kp} / \mathrm{cm}^{2}$ aceptables como valor mínimo. Los resultados pueden tener su explicación en el hecho de que, independientemente de haber cocido a temperaturas diferentes, los ladrillos rojos tienen un contenido en cuarzo algo más elevado, esta presencia se puede asociar a un mayor aporte de arena como desgrasante, lo que unido a

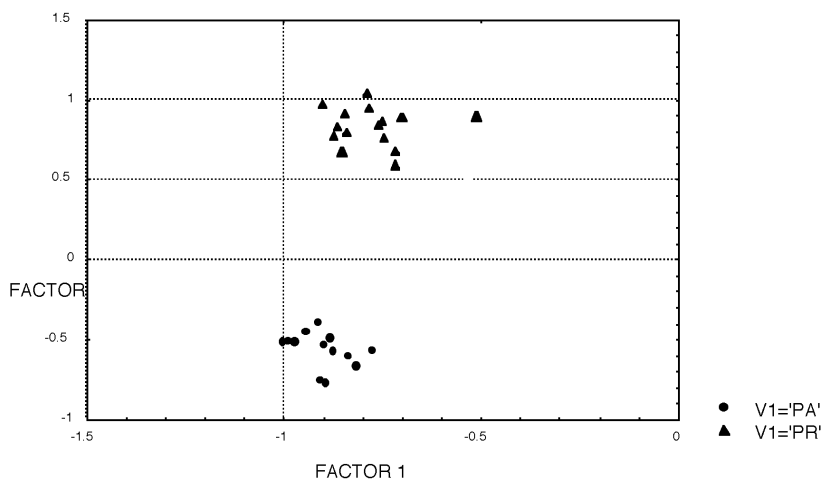

Fig. 2. Mapa de posición de puntos Factor 1 / Factor 2.

una reacción cerámica más completa puede contribuir a mejorar sus propiedades mecánicas (10). En la tabla 1 se expone el resumen de los resultados.

\subsection{Análisis químico.}

Los dos grupos de muestras procedentes de La Pañoleta, amarillo y rojo, no presentan diferencias significativas, lo cual era previsible, debido a que las características de las materias primas y condiciones de cocción son similares (tablas 2 y 3 ).

La diferencia más notable entre ambos grupos, es el mayor contenido en hierro de las muestras rojas, lo cual era perfectamente esperable, puesto que este componente es el causante de la coloración de estas piezas. Otra diferencia entre las piezas amarillas y rojas es la mayor P.F. de las muestras PR, lo que tiene su origen, como se indica en la discusión de resultados del análisis mineralógico, en la menor temperatura de cocción alcanzada.

TABLA II

ANÁLISIS QUÍMICO (\%) MUESTRAS PR

\begin{tabular}{|l|l|l|l|l|l|l|l|l|l|l|}
\hline & $\mathbf{S i O}_{2}$ & $\mathbf{A l}_{\mathbf{2}} \mathbf{O}_{3}$ & $\mathbf{F e}_{\mathbf{2}} \mathbf{O}_{3}$ & $\mathbf{C a O}$ & $\mathbf{M g O}$ & $\mathbf{T i O}_{2}$ & $\mathbf{N a}_{2} \mathbf{O}_{2}$ & $\mathbf{K}_{2} \mathbf{O}$ & $\mathbf{S O}_{3}$ & $\mathbf{P . F}$ \\
\hline PR1 & 59,78 & 9,04 & 7,92 & 13,52 & 1,93 & 0,08 & 1,54 & 1,93 & 0,11 & 3,45 \\
\hline PR2 & 60,11 & 11,20 & 7,73 & 13,63 & 2,03 & 0,07 & 1,42 & 2,24 & 0,18 & 1,28 \\
\hline PR3 & 59,47 & 11,78 & 7,16 & 13,29 & 2,04 & 0,07 & 1,43 & 1,84 & 0,18 & 2,52 \\
\hline PR4 & 58,76 & 10,17 & 8,22 & 12,95 & 2,06 & 0,08 & 1,64 & 2,47 & 0,33 & 3,17 \\
\hline PR5 & 59,95 & 8,97 & 7,83 & 12,98 & 1,98 & 0,08 & 1,78 & 2,18 & 0,41 & 3,53 \\
\hline PR6 & 59,12 & 9,17 & 7,80 & 13,19 & 2,20 & 0,08 & 1,60 & 2,20 & 0,67 & 3,48 \\
\hline PR7 & 59,91 & 4,48 & 8,23 & 13,37 & 2,06 & 0,08 & 1,44 & 2,26 & 0,49 & 7,13 \\
\hline PR8 & 59,47 & 10,62 & 7,81 & 12,94 & 2,05 & 0,09 & 1,64 & 1,85 & 0,27 & 2,63 \\
\hline PR9 & 56,98 & 8,14 & 7,85 & 13,42 & 1,89 & 0,07 & 1,24 & 2,27 & 0,33 & 7,60 \\
\hline PR10 & 60,28 & 8,25 & 8,42 & 12,84 & 2,21 & 0,10 & 1,60 & 2,21 & 0,26 & 3,44 \\
\hline PR11 & 60,57 & 8,42 & 8,34 & 12,81 & 2,03 & 0,07 & 1,42 & 2,03 & 0,18 & 3,75 \\
\hline PR12 & 60,33 & 9,08 & 8,20 & 13,14 & 2,05 & 0,07 & 1,64 & 2,05 & 0,15 & 3,06 \\
\hline PR13 & 57,49 & 11,59 & 8,17 & 12,86 & 2,04 & 0,08 & 1,63 & 1,84 & 0,57 & 3,47 \\
\hline PR14 & 59,71 & 10,06 & 7,66 & 12,77 & 1,96 & 0,08 & 1,57 & 2,16 & 0,14 & 3,44 \\
\hline PR15 & 59,19 & 10,14 & 8,32 & 13,27 & 1,98 & 0,09 & 1,58 & 2,38 & 0,15 & 2,69 \\
\hline
\end{tabular}


A partir del análisis estadístico factorial se pueden tratar de establecer parámetros que ayuden a discriminar entre los dos subgrupos a partir de la combinación de los resultados del análisis químico.

Con el fin de conseguir unos factores que alcancen una adecuada significación y que expliquen unos porcentajes de varianza elevados, tanto por separado, como en conjunto, se ha seleccionado una serie de parámetros que, en función de los factores de carga rotados, se han agrupado en tres factores, que tienen un significación suficientemente importante, considerando para su elección un factor de carga mínimo por encima del $80 \%$, obteniéndose un porcentaje total de varianza explicada del $85.5 \%$ (tabla 4).

Los factores que han resultado de esta operación han sido los siguientes:

- El factor 1 queda compuesto por silicio y calcio, ambos están relacionados entre sí, siendo los componentes que intervienen en las principales reacciones que se producen durante la cocción, dando lugar a silicatos cálcicos.

- El factor 2 agrupa sodio y potasio, son componentes que se presentan juntos formando parte de los feldespatos.

- El factor 3 se compone únicamente de hierro que, como se ha indicado, tiene su importancia como óxido que se modifica durante la cocción, siendo responsable de las alteraciones cromáticas, muy sensibles en el caso de estas piezas por el aclaramiento producido.

A continuación (Figuras 2 y 3) se exponen los mapas de posición de puntos en los que, con objeto de simplificar el análisis, se ha representado el factor 1 frente a los otros dos, en ellos se observa el grado de relación que, en función del análisis factorial, tienen las muestras y grupos de muestras entre sí. Con el fin de clarificar lo más posible estas representaciones, las muestras se han agrupado en las cinco áreas de procedencia ya referidas. De forma clara, el agrupamiento de las muestras es muy significativo, especialmente frente a los factores 2 y 3 , no así frente al 1, puesto que las piezas tienen unas características similares con respecto a los componentes que en él se agrupan.

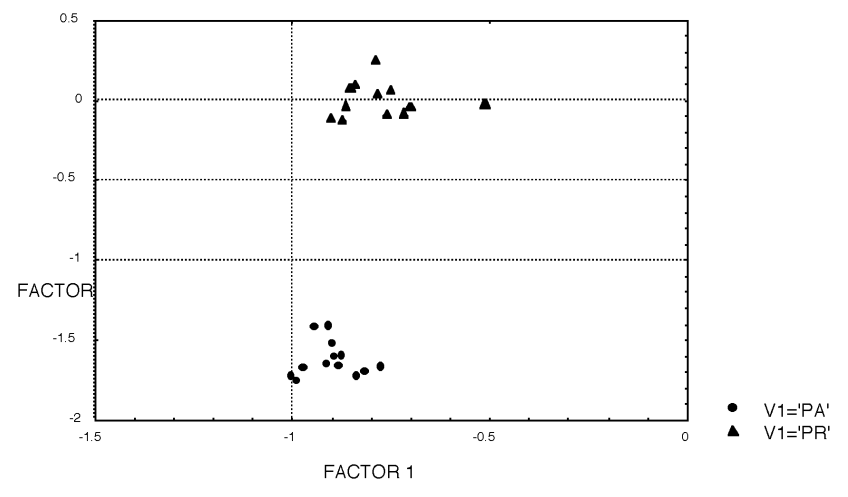

Fig. 3. Mapa de posición de puntos Factor 1 / Factor 3.

\subsection{Análisis mineralógico.}

Las muestras signadas como PA, de color amarillo no presentan importantes divergencias para las diversas fases, siendo destacable la ausencia total de calcita, lo que pone de manifiesto su descomposición completa durante la cocción.

El contenido en feldespatos es, en general, elevado, lo que resulta coherente con los también elevados porcentajes de sodio y potasio.

Son también altos los valores de gehlenita y aún mayores los de piroxeno, pudiéndose interpretar como diópsido (11). Teniendo en cuenta los porcentajes de magnesio obtenidos, la mayor presencia de piroxeno, es explicable a partir del análisis químico. En general estos resultados junto a la presencia generalizada de hematites, que se traduce en la presencia de zonas rojizas en el interior de las piezas, y de un silicato de calcio, que podría identificarse como kilchoanita o una estructura similar, permite suponer una temperatura de cocción alrededor de los $900^{\circ} \mathrm{C}$ (12).

TABLA 3

ANÁLISIS QUÍMICO (\%) MUESTRAS PA

\begin{tabular}{|lllllllllll|}
\hline & $\mathbf{S i O}_{2}$ & $\mathbf{F e}_{2} \mathbf{O}_{3}$ & $\mathbf{A l}_{2} \mathbf{O}_{3}$ & $\mathbf{C a O}$ & $\mathbf{M g O}$ & $\mathbf{T i O}_{2}$ & $\mathbf{N a}_{2} \mathbf{O}$ & $\mathbf{K}_{\mathbf{2}} \mathbf{O}^{2}$ & $\mathbf{S O}_{3}$ & $\mathbf{P . F}$ \\
\hline PA1 & 60,42 & 4,41 & 14,16 & 11,89 & 2,40 & 0,06 & 2,33 & 3,25 & 0,17 & 0,33 \\
\hline PA2 & 60,02 & 4,47 & 13,26 & 12,06 & 2,39 & 0,10 & 3,01 & 3,52 & 0,23 & 0,63 \\
\hline PA3 & 60,35 & 4,40 & 14,34 & 11,54 & 2,40 & 0,11 & 2,85 & 3,33 & 0,18 & 0,39 \\
\hline PA4 & 60,74 & 4,25 & 14,27 & 11,66 & 2,33 & 0,09 & 2,45 & 3,35 & 0,16 & 0,37 \\
\hline PA5 & 60,11 & 4,62 & 14,05 & 12,23 & 2,32 & 0,09 & 2,42 & 3,13 & 0,28 & 0,40 \\
\hline PA6 & 59,89 & 4,30 & 13,96 & 12,15 & 2,23 & 0,11 & 2,25 & 3,49 & 0,36 & 0,64 \\
\hline PA7 & 60,03 & 4,43 & 13,82 & 12,26 & 2,25 & 0,10 & 2,38 & 3,42 & 0,28 & 0,57 \\
\hline PA8 & 59,92 & 4,47 & 13,88 & 12,07 & 2,25 & 0,13 & 2,68 & 3,33 & 0,37 & 0,61 \\
\hline PA9 & 59,73 & 4,43 & 14,08 & 12,18 & 2,33 & 0,06 & 2,88 & 3,24 & 0,37 & 0,60 \\
\hline PA10 & 60,02 & 4,25 & 13,93 & 12,18 & 2,30 & 0,09 & 2,23 & 3,32 & 0,40 & 0,60 \\
\hline PA11 & 60,41 & 4,40 & 13,29 & 12,21 & 2,27 & 0,09 & 2,89 & 3,17 & 0,18 & 0,69 \\
\hline PA12 & 60,12 & 4,23 & 13,57 & 12,18 & 2,35 & 0,08 & 2,89 & 3,37 & 0,27 & 0,65 \\
\hline PA13 & 59,37 & 4,01 & 14,15 & 12,26 & 2,25 & 0,11 & 2,93 & 3,52 & 0,21 & 0,63 \\
\hline PA14 & 59,25 & 4,31 & 14,34 & 12,24 & 2,25 & 0,14 & 2,19 & 3,33 & 0,38 & 0,92 \\
\hline PA15 & 59,29 & 4,63 & 14,05 & 12,59 & 2,32 & 0,06 & 2,35 & 3,42 & 0,16 & 0,61 \\
\hline
\end{tabular}


TABLA 4

PORCENTAJE DE VARIANZA EXPLICADA POR LOS DISTINTOS FACTORES.

\begin{tabular}{|l|c|}
\hline FACTOR & VARIANZA EXPLICADA \\
\hline F1 & $35,0 \%$ \\
\hline F2 & $30,2 \%$ \\
\hline F3 & $20,3 \%$ \\
\hline TOTAL & $85,5 \%$ \\
\hline
\end{tabular}

$\mathrm{Al}$ igual que en otros grupos de muestras, la presencia de yeso en el análisis mineralógico está relacionada con la detección de sulfatos en el análisis químico, la presencia de anhidrita podría relacionarse con la presencia de yeso en las materias primas, el cual se deshidrataría durante la cocción $\left(330^{\circ} \mathrm{C}\right)$, pudiendo haberse rehidratado con posterioridad. También encontraría su justificación en la baja calidad de los combustibles empleados en los hornos, polvo de carbón.

Las muestras de color rojo tienen en conjunto una menor homogeneidad que las amarillas, resaltando especialmente entre ellas como excepciones las piezas 7 y 9 .

En los contenidos en cuarzo, destacan las dos muestras referidas, con unos valores superiores al resto. Del mismo modo, la calcita aparece de forma irregular en nueve de las muestras, con valores mínimos, salvo las piezas 7 y 9, cuyos contenidos son significativamente superiores, siendo también las que poseen la mayor P.F. $(7,13 \%$ y $7,60 \%)$.

Los feldespatos aparecen en igual medida para todas las muestras, con contenidos que oscilan en torno al $10 \%$.

Los silicatos cálcicos de alta temperatura presentan elevados contenidos, tanto de piroxeno como de gehlenita, siendo dichos contenidos similares, la mayor diferencia con el grupo amarillo es una presencia menor de piroxeno, lo que permite suponer una temperatura de cocción mínima alrededor de los $850^{\circ} \mathrm{C}$. En algunas muestras se ha detectado de manera esporádica un silicato cálcico que, como se indica anteriormente, se puede asociar a kilchoanita o a una fase similar. Es importante destacar que las muestras PR7 y PR9 no presentan ninguna de estas fases, lo que está en concordancia con los elevados contenidos de calcita ya referidos.

En estas muestras, tanto yeso como anhidrita, aparecen de forma generalizada, lo que, al igual que para el otro subgrupo

TABLA 5

ANÁLISIS MINERALÓGICO

\begin{tabular}{|c|c|c|c|c|c|c|c|c|c|c|}
\hline & $Q$ & $\mathrm{C}$ & $\mathbf{F}$ & $\mathbf{H}$ & D & G & $\mathbf{K}$ & $\mathbf{Y}$ & $\mathbf{A}$ & $\mathrm{T}^{\mathrm{o}}$ estimada \\
\hline PA1 & ++++ & & ++ & $\mathrm{t}$ & ++ & + & $\mathrm{t}$ & & $\mathrm{t}$ & 900 \\
\hline PA2 & +++++ & & ++ & $\mathrm{t}$ & ++ & ++ & $\mathrm{t}$ & $t$ & $\mathrm{t}$ & $850-900$ \\
\hline PA3 & ++++ & & ++ & $\mathrm{t}$ & +++ & ++ & & $\mathrm{t}$ & $\mathrm{t}$ & 900 \\
\hline PA4 & ++++ & & ++ & $\mathrm{t}$ & +++ & ++ & & & $\mathrm{t}$ & 900 \\
\hline PA5 & ++++ & & ++ & $\mathrm{t}$ & +++ & + & $\mathrm{t}$ & $\mathrm{t}$ & $\mathrm{t}$ & 900 \\
\hline PA6 & +++++ & & ++ & $\mathrm{t}$ & +++ & ++ & & $\mathrm{t}$ & $\mathrm{t}$ & 900 \\
\hline PA7 & ++++ & & ++ & & +++ & ++ & $\mathrm{t}$ & $\mathrm{t}$ & $\mathrm{t}$ & 900 \\
\hline PA8 & +++++ & & ++ & $\mathrm{t}$ & ++ & ++ & $\mathrm{t}$ & $\mathrm{t}$ & $\mathrm{t}$ & $850-900$ \\
\hline PA9 & +++++ & & ++ & $\mathrm{t}$ & ++ & ++ & $\mathrm{t}$ & $t$ & $\mathrm{t}$ & $850-900$ \\
\hline PA10 & ++++ & & ++ & & ++ & ++ & $\mathrm{t}$ & $\mathrm{t}$ & $\mathrm{t}$ & 900 \\
\hline PA11 & ++++ & & ++ & & +++ & ++ & $\mathrm{t}$ & $\mathrm{t}$ & $\mathrm{t}$ & 900 \\
\hline PA12 & ++++ & & ++ & $\mathrm{t}$ & ++ & + & $\mathrm{t}$ & $\mathrm{t}$ & & 900 \\
\hline PA13 & ++++ & & ++ & $\mathrm{t}$ & +++ & ++ & $\mathrm{t}$ & + & $\mathrm{t}$ & 900 \\
\hline PA14 & +++++ & & ++ & $\mathrm{t}$ & ++ & ++ & & $\mathrm{t}$ & $\mathrm{t}$ & $850-900$ \\
\hline PA15 & ++++ & & ++ & $\mathrm{t}$ & ++ & ++ & $\mathrm{t}$ & $\mathrm{t}$ & & $850-900$ \\
\hline PR1 & +++++ & & + & $\mathrm{t}$ & ++ & ++ & & $\mathrm{t}$ & $\mathrm{t}$ & 850 \\
\hline PR2 & +++++ & & + & $\mathrm{t}$ & ++ & ++ & & $\mathrm{t}$ & & 850 \\
\hline PR3 & +++++ & & & $\mathrm{t}$ & + & +++ & & & $\mathrm{t}$ & $800-850$ \\
\hline PR4 & ++++ & $\mathrm{t}$ & ++ & $\mathrm{t}$ & ++ & ++ & & $\mathrm{t}$ & $\mathrm{t}$ & 850 \\
\hline PR5 & +++++ & $\mathrm{t}$ & + & $\mathrm{t}$ & ++ & ++ & $\mathrm{t}$ & $\mathrm{t}$ & $\mathrm{t}$ & 850 \\
\hline PR6 & +++++ & & + & $\mathrm{t}$ & + & ++ & & $\mathrm{t}$ & + & $800-850$ \\
\hline PR7 & +++++ & ++ & + & & & & & & $t$ & 700 \\
\hline PR8 & ++++ & & ++ & & ++ & + & & $t$ & $\mathrm{t}$ & 900 \\
\hline PR9 & +++++ & ++ & + & $\mathrm{t}$ & & & & & $\mathrm{t}$ & 700 \\
\hline PR10 & +++++ & & + & $\mathrm{t}$ & + & ++ & $\mathrm{t}$ & $t$ & $t$ & $800-850$ \\
\hline PR11 & +++++ & + & + & $\mathrm{t}$ & + & ++ & & & $\mathrm{t}$ & $800-850$ \\
\hline PR12 & ++++ & + & ++ & $t$ & + & ++ & & & $\mathrm{t}$ & $800-850$ \\
\hline PR13 & ++++ & & ++ & + & + & ++ & $\mathrm{t}$ & $\mathrm{t}$ & + & $800-850$ \\
\hline PR14 & +++++ & + & ++ & $\mathrm{t}$ & + & ++ & & & $\mathrm{t}$ & $800-850$ \\
\hline PR15 & ++++ & $\mathrm{t}$ & ++ & $\mathrm{t}$ & ++ & ++ & $\mathrm{t}$ & $\mathrm{t}$ & $\mathrm{t}$ & 850 \\
\hline
\end{tabular}

Q: cuarzo, C: calcita, F: feldespatos, H: hematites, P: piroxeno, G: gehlenita, K: silicato de calcio, Y: yeso, A: anhidrita. $+++++($ muy abundante), ++++(abundante), +++(presente), $++($ escaso $),+($ indicios $), \mathrm{t}$ (trazas). 
de muestras, concuerda con los elevados contenidos en sulfatos que presenta el análisis químico.

Por último, indicar la presencia de hematites en trece de las quince muestras, lo que resulta lógico teniendo en cuenta el importante contenido en óxido de hierro que se observa en el análisis químico. Esta presencia de hematites es la causa directa del color rojizo de estos ladrillos (13).

El conjunto de los resultados obtenidos a partir del evaluación de las muestras se recoge en la tabla 5, quedando los mismos expresado mediante rangos de detección.

\subsection{Análisis térmico.}

El perfil de las curvas de ATD de las muestras de ladrillo analizadas es muy suave y similar para todas las piezas, sin ningún efecto destacable que distorsione las mismas. Tan sólo aparecen efectos de carácter exotérmico de pequeña intensidad a partir de $\operatorname{los} 850^{\circ}-900^{\circ} \mathrm{C}$. Estos resultados confirman las previsiones realizadas a partir del análisis mineralógico para las temperaturas de cocción. La gráfica de TG no manifiesta pérdida de peso significativa durante el calentamiento, lo que concuerda con la mínima P.F. cuantificada en el análisis quími$\mathrm{co}$, con las excepciones de las muestras reseñadas como diferentes en este aspecto.

\section{CONCLUSIONES}

Todas las piezas presentan un porcentaje de absorción de agua por encima del límite admitido por la normativa vigente $(18 \%)$. Los valores de succión se encuentran por debajo del valor máximo admisible $\left(0.45 \mathrm{~g} / \mathrm{cm}^{2}\right)$. Ambas series de resultados son similares para los dos tipos descritos, siendo mayor la homogeneidad para las piezas tipo amarillo. La resistencia a compresión no resulta similar para los dos grupos siendo sensiblemente mayor para las piezas tipo rojo lo que es asociable a un mayor desarrollo durante la cocción.

El contenido de calcio se puede asociar a la presencia de silicatos cálcicos, siendo muy bajo el contenido en carbonatos puesto que al ser piezas de moderna factura no han sido susceptibles de sufrir procesos de recarbonatación (14).

La composición química es similar para los dos subgrupos de muestras, presentando una homogeneidad muy importante; ahora bien, el análisis factorial mediante el tratamiento estadístico de los parámetros correspondientes a los tres factores antes señalados permite hacer una discriminación entre ambos.

El factor 2, correspondiente a los óxidos de sodio y potasio, distingue de manera clara los subgrupos, lo que resulta coherente con las sensibles diferencias apreciadas en los porcentajes de dichos analitos, así como de feldespatos, fase mineral asociada a dichos componentes.

El factor 3, asociado al óxido de hierro, también discrimina claramente los dos grupos, estando ello plenamente justificado por la diferencia de color, directamente ligada a la presencia de dicho óxido, de tal manera que la presencia de este analito permite establecer una clara distinción entre los dos grupos, lo que unido a la influencia de los factores 1 y 2 posibilita un análisis factorial con plena significación.

Los contenidos en piroxeno y gehlenita se encuentran en proporciones inversas, esto es, el diópsido es más abundante en el subgrupo amarillo, y la gehlenita en el rojo. A partir de ello se puede deducir una mayor temperatura de cocción en las piezas amarillas, en torno a $\operatorname{los} 900^{\circ} \mathrm{C}$, mientras que las rojas habrían alcanzado aproximadamente unos $850^{\circ} \mathrm{C}$. Esta determinación se hace a partir de que el piroxeno aparece a mayor temperatura $\left(\geq 850^{\circ} \mathrm{C}\right)$ que la gehlenita $\left(\geq 800^{\circ} \mathrm{C}\right)$, y su formación conllevaría la parcial desaparición de esta última. Esta posibilidad se ve reforzada con los resultados obtenidos en el análisis térmico diferencial, en la Tabla 5 se incluyen los rangos estimados para las temperaturas de cocción de cada una de las muestras.

\section{AGRADECIMIENTOS.}

Los autores del presente trabajo quieren expresar su agradecimiento al Grupo de Mineralogía Aplicada del Dpto. de Cristalografía, Mineralogía y Química Agrícola de la Universidad de Sevilla por su colaboración en la realización de los análisis mineralógicos y descripción litológica de la zona y al instituto de Ciencia de los Materiales de Sevilla por su colaboración en la realización de ATD y TG.

\section{BIBLIOGRAFÍA.}

1. F. González y col.. «Firing transformations of mixtures of clays containing illite, kaolinite and calcium carbonate used by ornamental tile industries», Applied Clay Sc., 5, 361-375 (1990).

2. E.Galán, J.L. Pérez y col..«Geología de Sevilla y alrededores», pp. 63-78, Ayto. de Sevilla, Sevilla (España), 1989.

3. G. García-Ramos, F. González-García, J.L. Pérez-Rodríguez, « Studio mineralogico e tecnologico delle argille ceramiche del Sud.Ovest della Spagna (Andalusia e Estremadura)» («Mineralogical and technological study of the ceramic clays from the South-West of Spain (Andalusia and Extremadura)»), Ceramica Infromazione, 149, 688-705 (1978).

4. F. González-García, G. García-Ramos, «Arcillas cerámicas de Andalucía. III.Yacimientos terciarios de la margen derecha del Guadalquivir en la provincia de Sevilla», Bol. Soc. Esp. Cerámica y Vidrio, 5 (2), 229-245 (1966).

5.F. González-García, G. García-Ramos, «Arcillas cerámicas de Andalucía. I.Yacimientos de las vegas del Guadalquivir y Corbones en la provincia de Sevilla», Bol. Soc. Esp. Cerámica y Vidrio, 3 (5), 481-502 (1964).

6. F. González-García y col.. «Estudio de algunas margas y otros materiales análogos de Andalucía occidental», pp. 53-58, en Cerámica y Vidrio 92 (Resúmenes del XXXII Cong. Nac. De Cerámica y Vidrio), Eds. Científicos F. Capel, A. Caballero y J.Ma . Rincón, Soc. Esp. Cerámica y Vidrio, Madrid, 1992.

7. F. González-García, G. García-Ramos, V. Romero, M. González, «Arcillas empleadas en la fabricación del azulejo artístico de Sevilla y transformaciones por cocción. I. Materiales que contienen carbonato de calcio», Bol. Soc. Esp. Cerámica y Vidrio, 27 (2), 215-223 (1988).

8. V. Romero, A. Justo, G. García, «Arcillas del valle del Guadalquivir empleadas como soporte de azulejos en la industria sevillana. I», Bol. Soc. Esp. Cerámica y Vidrio, 15 (3), 163-169 (1976).

9. A. Martín. «Ensayos y experiencias de alteración en la conservación de obras de piedra de interés histórico artístico», pp. 279-281, Fund. Areces, Madrid (España), 1990.

10. G. Fazio y col.. «The Altinelle in Venice». G.P. Newsletter 4, 20-26 (1983).

11. M. Vendrell-Saz, T. Pradell-Clara. «Caracterización química y mineralógica de la cerámica sigillata tardía producida en Clunia (S.IV al VII)». Bol. Soc. Esp. de Mineralogía 15, 31-40 (1992).

12. C. Dell'Aquila, R. Laviano, A. Viceconte, F. Vurro. «Mineralogical and chemical characterization of clay bodies», pp. 271-282, en Fourth Euro-Ceramic vol.14: The Cultural Ceramic Heritage, $3^{\text {rd }}$ European Meeting on Ancient Ceramics, Riccione (Italia), 1995. Ed. B. Fabbri, Societa'Ceramica Italiana.

13. R. Kreimeyer. «Some notes on the firing colour of clays bricks». Applied Clay Sc. 2, 175-183 (1987).

14. B. Fabbri, V. Fassina, A. Rattazzi, D. Salvioni. «The majolica flooring of San Sebastiano in Venice», pp. 383-392, en Fourth Euro-Ceramic vol.14: The Cultural Ceramic Heritage, $3^{\text {rd }}$ European Meeting on Ancient Ceramics, Riccione (Italia), 1995. Ed. B. Fabbri, Societa'Ceramica Italiana. 